\title{
Intelligent Three-Dimensional Reconstruction Algorithm-Based X-Ray Film for Analysis of the Changes in Sagittal Parameters and Curative Effect of Anterior Cervical Surgery
}

\author{
Zhengyu Liu $\mathbb{D}$, Qingming Zhang $\mathbb{D}$, Juyong Wang $\mathbb{D}$, and Shibao Lu $\mathbb{D}$ \\ Department of Orthopedics, Xuanwu Hospital, Capital Medical University, No. 45 Changchun Street, Xicheng District, \\ Beijing 100053, China \\ Correspondence should be addressed to Shibao Lu; 201998000121@hceb.edu.cn
}

Received 19 May 2021; Accepted 11 July 2021; Published 20 July 2021

Academic Editor: Gustavo Ramirez

Copyright (c) 2021 Zhengyu Liu et al. This is an open access article distributed under the Creative Commons Attribution License, which permits unrestricted use, distribution, and reproduction in any medium, provided the original work is properly cited.

In this paper, the change characteristics of sagittal parameters of the patient's body before and after anterior cervical surgery and rehabilitation effect were analyzed. The rotation transformation and perspective projection transformation were applied to construct a positive and lateral position (PLP) matching-based three-dimensional reconstruction (PLP-3DR) algorithm, which was compared with the marching cubes (MC) algorithm and gravity point (GP) algorithm. PLP-3DR was adopted in X-ray image diagnosis of 124 cervical spondylosis patients. Results showed that sensitivity, specificity, and accuracy of PLP-3DR were markedly higher than those of MC and GP $(P<0.05)$. The postoperative C2-7 Cobbs's angle $(\mathrm{C} 2-7 \mathrm{Cobb})\left(11.27 \pm 8.41^{\circ}\right)$ was smaller than that before the surgery $\left(15.03 \pm 7.39^{\circ}\right)(P<0.05)$, while the postoperative cervical sagittal vertical axis $(\mathrm{cSVA})(21.33 \pm 10.38 \mathrm{~mm})$ and Japanese Orthopaedic Association (JOA) scores (16.95 \pm 6.07 points) were greater than those before the surgery $(14.66 \pm 9.68 \mathrm{~mm}$ and $11.39 \pm 4.28$ points $)(P<0.05)$. Besides, the improvement rate of patients from the cSVA $>25 \mathrm{~mm}$ group $(68.31 \%)$ was greater than the rate of the cSVA $<15 \mathrm{~mm}$ group (45.88\%) and the cSVA within $15-25 \mathrm{~mm}$ group (47.29\%) $(P<0.05)$. In conclusion, PLP-3DR could effectively improve the diagnostic effect of spine X-ray film images, with high sensitivity and specificity. Anterior cervical surgery had a good curative effect for patients with cervical spondylosis and was closely related to cervical parameters (cSVA, T1 slope, and C2-7Cobb). In addition, patients with cSVA $>25 \mathrm{~mm}$ could have the best postoperative rehabilitation effect.

\section{Introduction}

The spine, as a critical part of the human skeleton, is located on the back of the human body. It mainly includes the thoracic, sacral, coccyx, cervical, and lumbar bones, which can help the human body to walk and work normally $[1,2]$. In the process of clinical understanding of the balance of the spine, it is found that the imbalance of the spine has a relatively intuitive impact on clinical symptoms. If the spine is diseased, it will cause ankylosing cervical spine inflammation, lumbar disc prominence, and other diseases [3,4]. Therefore, cervical spondylosis has always been the focus of clinical attention, including cervical osteoporosis, sympathetic nerve compression, and cervical degenerative diseases [5]. Anterior cervical surgery is a common method with good curative effect and relatively safe treatment for cervical degenerative diseases, trauma, tumor, inflammation, and deformity. However, the surgery is difficult, with high risk $[6,7]$. Therefore, the transformation of sagittal parameters before and after clinical anterior cervical spine surgery is a topic worth investigating.

With the continuous progress and development of computer technology, virtual reality technology, and image processing technology, three-dimensional visualization of medical images has been extensively applied in biomedical engineering, such as medical teaching, radiation therapy, medical orthopedics, and virtual surgery $[2,8]$. Image threedimensional reconstruction generally includes surface rendering and volume rendering. Surface rendering mainly uses volume data to construct intermediate geometric units to 
simulate the surface information of the target object, while volume rendering does not need to construct intermediate geometric units and can directly project volume data to the display screen, so as to obtain three-dimensional images [9]. Therefore, it is very crucial to select the appropriate construction technology to process medical images. The clinical diagnosis of spinal diseases cannot be separated from the participation of imaging, such as plain X-ray film, magnetic resonance imaging (MRI), and computed tomography (CT). CT images can display high-resolution images of the human body and the location of the lesions, but the three-dimensional reconstruction of CT images requires hundreds of two-dimensional images of the spine, and the operation efficiency is low [10]. MRI images have a good display effect on human soft tissues and have the advantages of multiparameters, high resolution, and high diagnostic sensitivity, but the operation is very complicated, with expensive cost, and not suitable for general application [11]. An X-ray film is an image in the traditional sense. In the acquisition of spine images, the image quality will be reduced due to the interference of noise, which will affect the reconstructed data. Therefore, it is very critical to adopt appropriate three-dimensional reconstruction algorithm.

To sum up, the three-dimensional reconstruction of medical images plays an important role in imaging diagnosis. Based on this, the rotation transformation and perspective projection transformation were first applied to construct the PLP-3DR algorithm. Then, MC and GP were introduced to compare with PLP-3DR. Thus, PLP-3DR was applied to the X-ray film image diagnosis of 124 patients with cervical spondylosis, thereby comprehensively evaluating the changes in the sagittal parameters of the patients before and after anterior cervical surgery and the rehabilitation effect.

\section{Materials and Methods}

2.1. Selection of Research Samples. In this study, 124 patients with cervical spondylosis were selected as the research objects, who were admitted to hospital from January 10, 2018, to January 11, 2020, and were treated with anterior surgeries. The surgical protocols included anterior cervical corpectomy decompression and fusion (ACCF), anterior cervical discectomy and fusion (ACDF), and a combination of ACCF and ACDF. There were 76 males and 48 females, aged 20-65 years. This study had been approved by the medical ethics committee of the hospital, and the patients and their family members had understood the situation of this study and signed the informed consent forms.

The criteria for inclusion were defined to include patients who had complete basic clinical data, had underwent anterior cervical surgeries for more than 1 year, had radicular symptoms such as neck, shoulder, and arm pain in the cervical spine, suffered from limb motor dysfunction, and were older than 18 years of age.

The criteria for exclusion were defined to include patients who had a history of cervical spine trauma, had a history of cervical spine surgery, suffered from cervical congenital malformations, had received anterior cervical spine surgery combined with posterior surgery, withdrew from the experiment for personal reasons, and were younger than 66 years of age.

2.2. X-Ray Imaging Examination. X-ray films were employed to perform imaging examinations on the patients 1 week before surgery and during the postoperative review. When shooting, the patient should stand naturally, keep the line of sight straight ahead, and hang the upper limbs naturally on both sides of the body, thereby clearly photographing the upper edge of the $\mathrm{C} 7$ vertebral body and the upper edge of the sternum stem. After the shooting was completed, 2 senior orthopedic resident doctors applied Surgimap measuring software to detect the parameters of the image map, including C2-7Cobb, T1 slope, thoracic inlet angle (TIA), neck tilt (NT), and cSVA.

\subsection{Three-Dimensional Reconstruction Algorithm Based on} the Positive and Lateral Position Matching. A spine X-ray plain film is easily affected by exposure, which greatly reduces the display quality. Therefore, the histogram equalization was first employed to preprocess the original image. This method can make the output image have the same pixel points in each gray level through the gray point operation [12], which can be expressed as follows:

$$
W=\frac{L_{\max }}{S} \sum_{r=0}^{e} M(r) .
$$

In (1), $W$ stood for the gray level after transformation, $e$ represented the gray level after normalization, $S$ expressed the area of the original image, $M(i)$ indicated the $i$-th gray level, and $L_{\max }$ meant the maximum gray level. The basic principle of PLP-3DR was as follows. Each spine model received rotation transformation and perspective projection transformation to obtain a projection map similar to the original image spine block. Then, the projection map was matched with the spine block, and the parameters were adjusted to make the matching successful. The scoliosis spine model was reconstructed according to the successfully matched three-dimensional parameters. A simple one-point perspective projection was adopted in this study to extract the three-dimensional parameters. Assuming that the coordinates of the viewpoint in a three-dimensional space were $\left(s_{x}, s_{y}, s_{z}\right)$ and the coordinates of a point on the three-dimensional model were $\left(p_{x}, p_{y}, p_{z}\right)$, the coordinates of the point projected onto the coordinate plane were $\left(u_{x}, u_{y}, u_{z}\right)$ that could be expressed in the following equations:

$$
\begin{aligned}
& u_{x}=\frac{s_{x} p_{z}-s_{z} p_{x}}{p_{z}-s_{z}}, \\
& u_{y}=\frac{s_{y} p_{z}-s_{z} p_{y}}{p_{z}-s_{z}}, \\
& u_{z}=0 .
\end{aligned}
$$

Then, it was shown that the perspective of the positive phase perspective view was on the $Y$-axis, and the 
perspective plane was the $X O Z$ plane according to the coordinate relationship. Besides, the perspective view of the lateral perspective view was on the $X$-axis, and the perspective plane was the $Y O Z$ plane. Thus, the coordinate value of any point in the three-dimensional model on the projection plane after the projection transformation was as follows:

$$
\begin{aligned}
& x=x_{s}+\left(x_{i}-x_{s}\right) d, \\
& y=y_{s}+\left(y_{i}-y_{s}\right) d, \\
& z=z_{s}+\left(z_{i}-z_{s}\right) d .
\end{aligned}
$$

In equations (5)-(7), $(x, y, z)$ stood for the coordinate value of any point in the three-dimensional model on the projection plane after projection transformation, $\left(x_{s}, y_{s}, z_{s}\right)$ represented the coordinate of the viewpoint, and $\left(x_{i}, y_{i}, z_{i}\right)$ expressed any point in the three-dimensional model. Therefore, when the viewpoint coordinates were on the $Y$ axis and the perspective plane was the $X O Z$ plane, the following equation could be obtained:

$$
d=\frac{y_{s}}{y_{s}-y_{i}} .
$$

Equations (9) and (10) could be attained by substituting $d$ into equations (5)-(7).

$$
\begin{aligned}
& x=\frac{x_{i} y_{s}-x_{s} y_{i}}{y_{s}-y_{i}}, \\
& z=\frac{z_{i} y_{s}-z_{s} y_{i}}{y_{s}-y_{i}} .
\end{aligned}
$$

Similarly, equation (11) could be obtained when the viewpoint coordinates were on the $X$-axis and the perspective plane was the $X O Z$ plane.

$$
d=\frac{x_{s}}{x_{s}-x_{i}},
$$

and $d$ was substituted into equations (5)-(7) to get the following equations:

$$
\begin{aligned}
& y=\frac{y_{i} x_{s}-x_{i} y_{s}}{x_{s}-x_{i}}, \\
& z=\frac{z_{i} x_{s}-z_{s} x_{i}}{x_{s}-x_{i}} .
\end{aligned}
$$

Therefore, substituting the obtained three-dimensional data coordinate points of the spine model into the abovementioned equations could give the expression of the positive and lateral surfaces of the spine model on the two-dimensional platform and then construct a three-dimensional X-ray image. In addition, the algorithm based on the positive and lateral position matching in this study was set as PLP-3DR.

2.4. Algorithm Evaluation Indicators. MC algorithm [13] and GP algorithm [14] were introduced to compare with the PLP-3DR algorithm proposed in this paper. The accuracy, sensitivity, and specificity were selected as the evaluation indicators of algorithm performance, which were expressed as follows:

$$
\begin{aligned}
& \text { Acc }=\left(\frac{F_{\text {True }}}{\text { Total }}\right) \times 100 \%, \\
& \text { Sen }=\left[\frac{\mathrm{TP}}{(\mathrm{TP}+\mathrm{FN})}\right] \times 100 \%, \\
& \text { Spe }=\left[\frac{\mathrm{TN}}{(\mathrm{TN}+\mathrm{FP})}\right] \times 100 \% .
\end{aligned}
$$

In equations (13)-(15), $F_{\text {True }}$, Total, TP, TN, FP, and FN stood for the number of cases with correct predictions, the total number of cases, true positive, true negative, false positive, and false negative, respectively.

2.5. Observation Indicators. The basic information of patients was recorded, such as age, gender, surgical method, and X-ray image data. They were rolled into 3 groups based on $\mathrm{cSVA}=15 \mathrm{~mm} / 25 \mathrm{~mm}$ as the dividing line, namely, the cSVA $<15 \mathrm{~mm}$ group, the cSVA within $15-25 \mathrm{~mm}$ group, and the cSVA $>25 \mathrm{~mm}$ group. Furthermore, the cervical sagittal parameters of patients should be recorded, such as C2-7Cobb, T1 slope, TIA, and NT. The JOA scoring was adopted to evaluate the efficacy of surgery and calculate its improvement rate. Furthermore, the calculation equation was Improvement rate $=\left(\mathrm{JOA}_{\text {postoperative }}-\mathrm{JOA}_{\text {Preoperative }}\right) /$ $\left(17-\mathrm{JOA}_{\text {Preoperative }}\right) \times 100 \%$. In addition, the indicator data of MC, GP, and PLP algorithms were calculated, including the accuracy, sensitivity, and specificity.

2.6. Statistical Methods. The data processing in this study was analyzed by SPSS19.0 version statistical software, the measurement data were expressed as mean \pm standard deviation $(\bar{x} \pm s)$, and the count data were represented by percentage (\%). The sensitivity, specificity, and accuracy of PLP-3DR, MC, and GP algorithms were for pairwise comparison, which were analyzed by analysis of variance. The improvement rate, C2-7Cobb, JOA scores, T1 slope, TIA, and NT of patients from the cSVA $>25 \mathrm{~mm}$ group, cSVA within $15-25 \mathrm{~mm}$ group, and the cSVA $<15 \mathrm{~mm}$ group were compared and detected by the paired $t$-test. In addition, $P<0.05$ meant that the difference was statistically substantial.

\section{Results}

3.1. Comparison on Noise Reduction Performance of Three Algorithms. Figure 1 indicates the comparison on the sensitivity, specificity, and accuracy of the three algorithms. It was found that the sensitivity, specificity, and accuracy of PLP-3DR were obviously greater than those of MC and GP, and the difference was statistically marked $(P<0.05)$. However, the sensitivity, specificity, and accuracy of MC and GP had no statistically substantial difference $(P>0.05)$. 


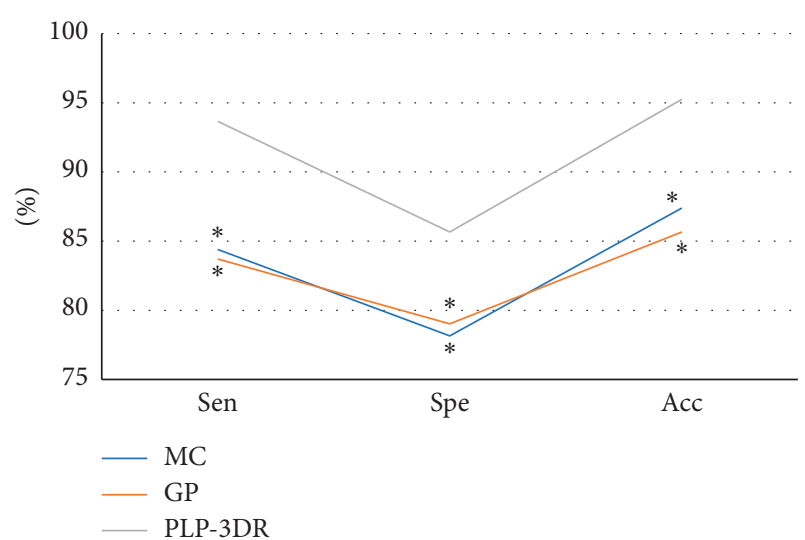

FIGURE 1: Comparison on the sensitivity, accuracy, and specificity of the three algorithms (note: ${ }^{*}$ meant that the difference was statistically substantial compared with PLP-3DR $(P<0.05))$.

3.2. Descriptive Statistics on Basic Data of All Patients. Figure 2 showed the age distribution of all patients. It revealed that the proportion of patients older than 50 years (57\%) was the highest, followed by the proportion of patients aged $40-50$ years $(33 \%)$, and the proportion of patients $20-40$ years of age was the least (10\%). Figure 3 indicated the proportion of patients with surgical methods. It demonstrated that the proportion of patients undergoing anterior cervical corpectomy and fusion (ACCF) surgery was the highest $(47.43 \%)$, followed by the proportion of patients with anterior cervical decompression and fusion (ACDF) surgery (39.51\%), and the proportion of patients receiving ACCF + ACDF combined surgery was the least (13.06\%).

3.3. X-Ray Manifestations of Some Patients. Figure 4 shows an X-ray film image of the spine of a male patient (aged 59 years). It was found that the shape of multiple vertebral bodies was irregular, the anterior upper edge was compressed, and the intervertebral space was narrowed, which could be diagnosed as spinal tuberculosis. Figure 5 shows an $\mathrm{X}$-ray film image of a female patient's spine, with an age of 35 years. It showed that there was a soft tissue-like density of the lesion, no dead bones, and clear edges that presented irregular worm-eaten damage or a saw-like appearance. In the later stage of sclerosis, bone spurs were formed by hyperplasia, presenting as a bird beak shape to extend outward or adjacent to the vertebral body margin, so as to form the bone bridge. The lesion in the center of the vertebral body rapidly hardened, without deep bone destruction defects, and was gradually replaced by new bone, without signs of vertebral compression.

3.4. Analysis Results of Overall Data of All Patients. The C27Cobb, cSVA, and JOA scores of patients were compared before and after surgery, and the results are shown in Figure 6. The C2-7Cobb before surgery was $15.03 \pm 7.39^{\circ}$, cSVA was $14.66 \pm 9.68 \mathrm{~mm}$, and JOA score was $11.39 \pm 4.28$ points. Besides, the C2-7Cobb, cSVA, and JOA score after surgery were $11.27 \pm 8.41^{\circ}, \quad 21.33 \pm 10.38 \mathrm{~mm}$, and
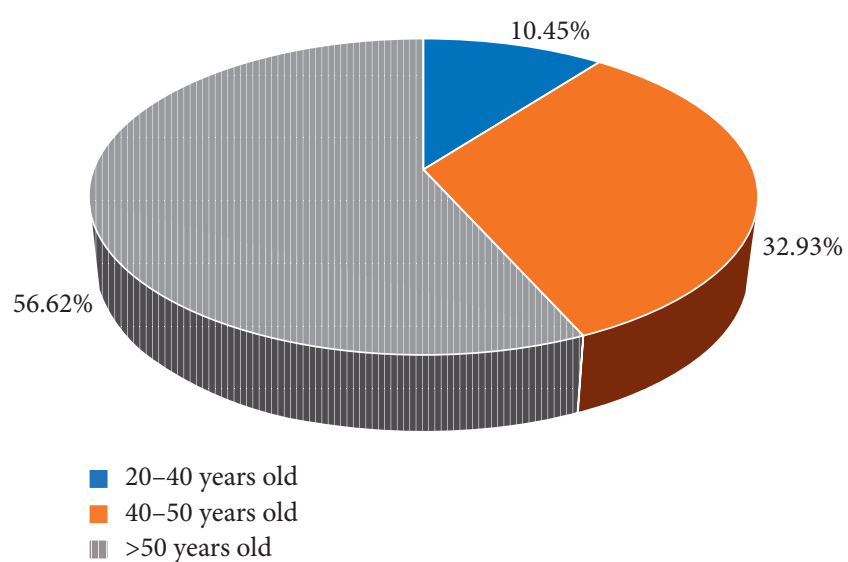

Figure 2: Age distribution of all the patients.

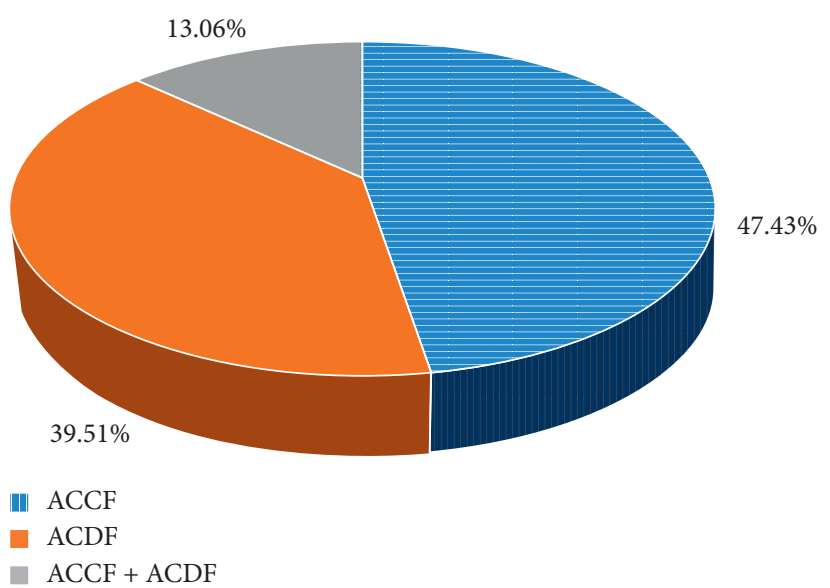

Figure 3: Proportion of patients with surgical methods.

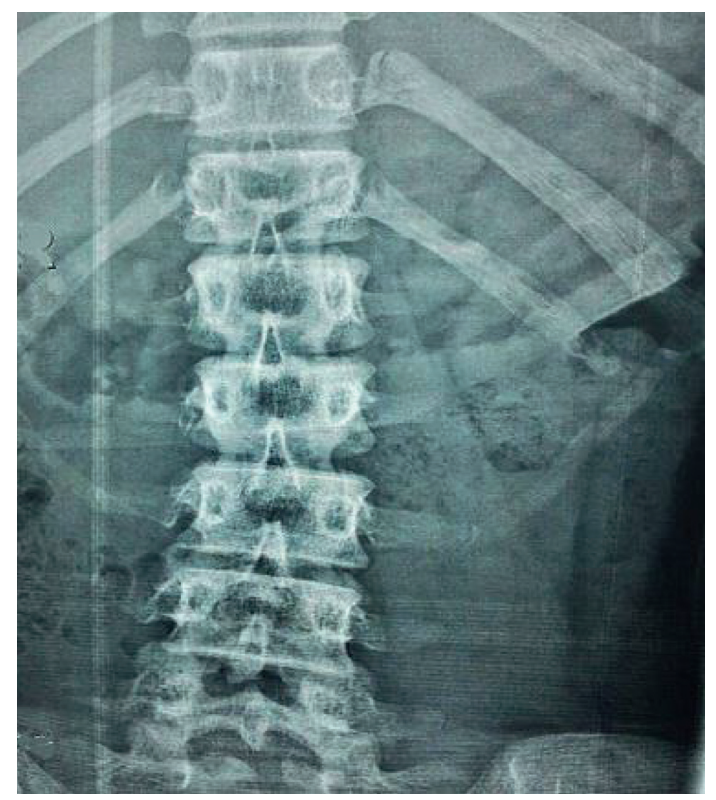

FIgURE 4: An X-ray image of a male patient's spine (aged 59 years).

$16.95 \pm 6.07$ points in turn. Thus, the C2-7Cobb after surgery was sharply smaller than that before surgery, with a statistically substantial difference $(P<0.05)$, while the cSVA 


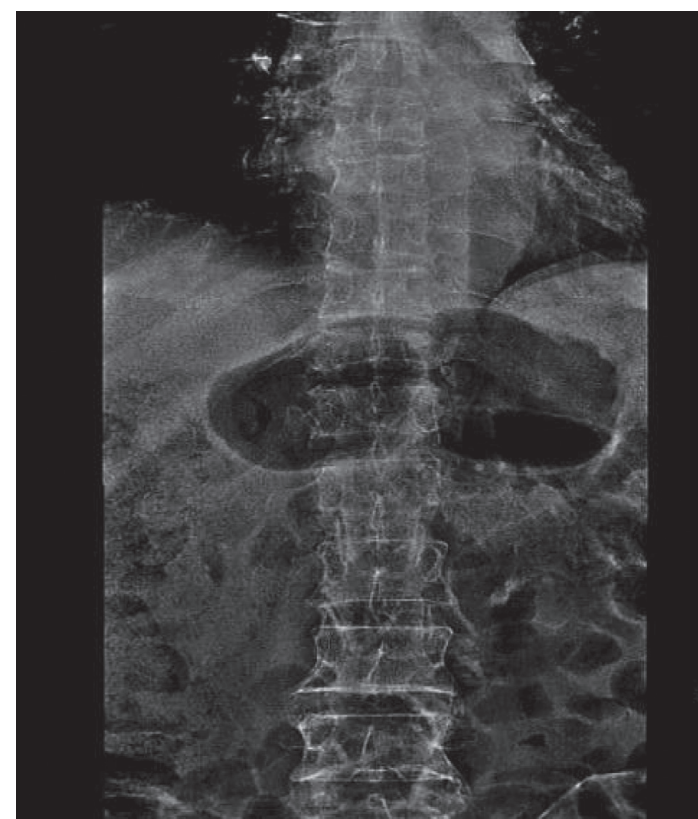

Figure 5: An X-ray film image of a female patient's spine (aged 35 years).

and JOA score after surgery were greater dramatically than those before surgery, showing a statistically marked difference $(P<0.05)$.

The T1 slope, TIA, and NT before and after surgery were compared, as shown in Figure 7. It was found that the T1 slope before surgery of patients was $24.38 \pm 8.31^{\circ}$, TIA was $79.32 \pm 11.25^{\circ}$, and NT was $58.03 \pm 12.31^{\circ}$. Furthermore, the T1 slope, TIA, and NT after surgery were $24.87 \pm 10.22^{\circ}$, $76.08 \pm 9.73^{\circ}$, and $56.88 \pm 10.44^{\circ}$ in sequence. However, the difference in T1 slope, TIA, and NT after surgery was not statistically obvious $(P>0.05)$.

3.5. Comparison on Improvement Rates of Patients from Different cSVA Groups after Surgery. Figure 8 revealed that there was a comparison on the improvement rates of patients from different cSVA groups after surgery. It showed that the improvement rate of patients from the cSVA $>25 \mathrm{~mm}$ group was $68.31 \%$, the improvement rate of the cSVA within $15-25 \mathrm{~mm}$ group was $47.29 \%$, and the improvement rate of the cSVA $<15 \mathrm{~mm}$ group was $45.88 \%$. Furthermore, the improvement rate of patients from the cSVA $>25 \mathrm{~mm}$ group increased markedly in contrast to the rate of the cSVA $<15 \mathrm{~mm}$ group and the cSVA within $15-25 \mathrm{~mm}$ group, and the difference was statistically remarkable $(P<0.05)$. However, there was no statistically obvious difference in the improvement rates of patients from the cSVA $<15 \mathrm{~mm}$ group and the cSVA within $15-25 \mathrm{~mm}$ group $(P>0.05)$.

3.6. Comparison on C2-7Cobb and JOA Scores of Patients from Different cSVA Groups after Surgery. Figure 9 shows the comparison results of $\mathrm{C} 2-7 \mathrm{Cobb}$ and JOA scores of patients from different cSVA groups after surgery. In the cSVA
$>25 \mathrm{~mm}$ group, the $\mathrm{C} 2-7 \mathrm{Cobb}$ was $6.88 \pm 4.27^{\circ}$ and the JOA score was $16.85 \pm 5.73$ points. In the cSVA within $15-25 \mathrm{~mm}$ group, the $\mathrm{C} 2-7 \mathrm{Cobb}$ and JOA score were $11.18 \pm 3.85^{\circ}$ and $16.32 \pm 5.38$ points, respectively. In addition, the $\mathrm{C} 2-7 \mathrm{Cobb}$ of patients from the cSVA $<15 \mathrm{~mm}$ group was $11.46 \pm 5.03^{\circ}$, and the JOA score was $16.49 \pm 6.02$ points. The C2-7Cobb of patients from the cSVA $>25 \mathrm{~mm}$ group was steeply lower than that of the CSVA $<15 \mathrm{~mm}$ group and the CSVA within $15-25 \mathrm{~mm}$ group, and the difference was statistically significant $(P<0.05)$. However, the $\mathrm{C} 2-7 \mathrm{Cobb}$ of patients from the cSVA $<15 \mathrm{~mm}$ group and the cSVA within $15-25 \mathrm{~mm}$ group was not statistically different $(P>0.05)$. Moreover, there was no statistically substantial difference in JOA scores of patients from the three groups $(P>0.05)$.

3.7. Comparison on T1 Slope, TIA, and NT of Patients from Different cSVA Groups after Surgery. The T1 slope, NT, and TIA of patients from different cSVA groups after surgery were compared, and the results are presented in Figure 10. It was found that the T1 slope, TIA, and NT of patients from the cSVA $>25 \mathrm{~mm}$ group were $30.51 \pm 8.95^{\circ}, 83.27 \pm 15.71^{\circ}$, and $61.04 \pm 11.23^{\circ}$, respectively. The T1 slope of patients from the cSVA within $15-25 \mathrm{~mm}$ group was $22.81 \pm 8.62^{\circ}$, TIA was $58.45 \pm 14.08^{\circ}$, and NT was $57.36 \pm 10.01^{\circ}$. In the cSVA $<15 \mathrm{~mm}$ group, the T1 slope was $22.45 \pm 7.29^{\circ}$, TIA was $55.21 \pm 11.42^{\circ}$, and NT was $56.24 \pm 12.43^{\circ}$. Thus, the T1 slope of patients from the cSVA $>25 \mathrm{~mm}$ group was greater hugely than that of the cSVA $<15 \mathrm{~mm}$ group and the cSVA within $15-25 \mathrm{~mm}$ group, and there was a statistically huge difference $(P<0.05)$. However, the difference in TIA and NT of patients from the cSVA $>25 \mathrm{~mm}$ group, the cSVA $<15 \mathrm{~mm}$ group, and the cSVA within $15-25 \mathrm{~mm}$ group was not statistically substantial $(P>0.05)$.

\section{Discussion}

The cervical spine is an important joint that connects the human skull to the trunk, which plays a prominent role in daily visual field adjustment and various complex sports. In anterior cervical surgery, the balance adjustment of the spine sagittal position is also an indispensable step. Therefore, it is clinically guiding significance to explore the changes of sagittal parameters in patients with cervical anterior surgery [15]. The PLP-3DR was established in this study by applying rotation transformation and perspective projection transformation. Moreover, MC and GP were introduced to compare with PLP-3DR in terms of performance. First of all, it was found that the sensitivity, specificity, and accuracy of PLP-3DR rose markedly compared with MC and GP $(P<0.05)$, which was consistent with the research findings of Duan et al. [3], indicating that the proposed PLP-3DR in this study could effectively promote the diagnostic effect of spine lesions, and had high sensitivity and specificity. PLP-3DR was applied to the X-ray image diagnosis of 124 patients with cervical spondylosis. The results showed that the patients' C2-7Cobb after surgery was significantly smaller than that before surgery $(P<0.05)$. C2-7Cobb has the largest range of motion and has a critical regulatory effect in the cervical 


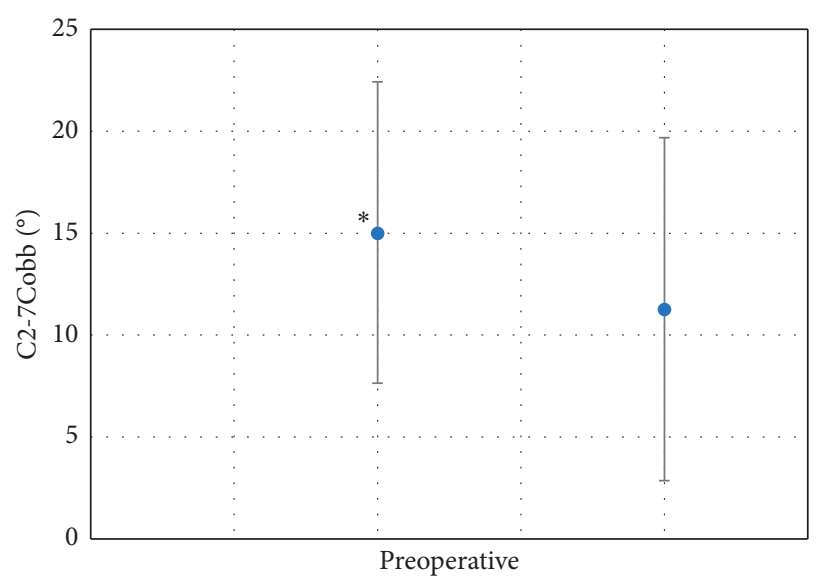

(a)

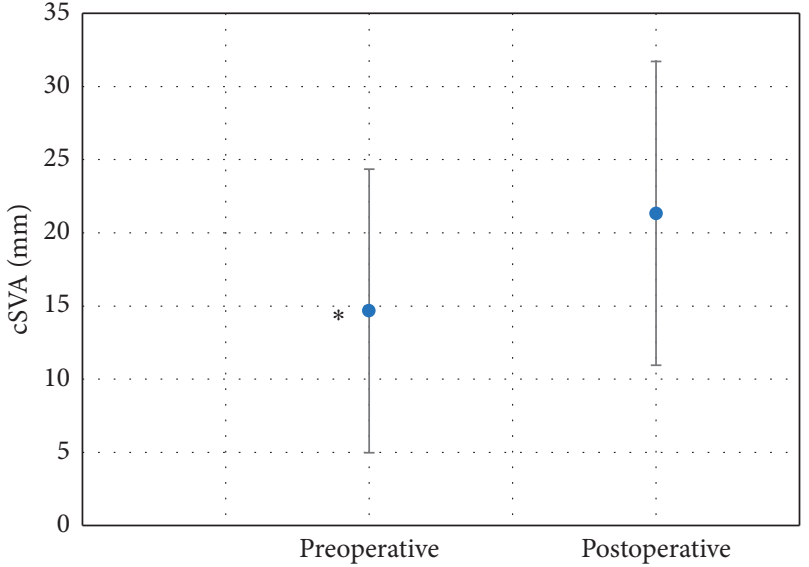

(b)

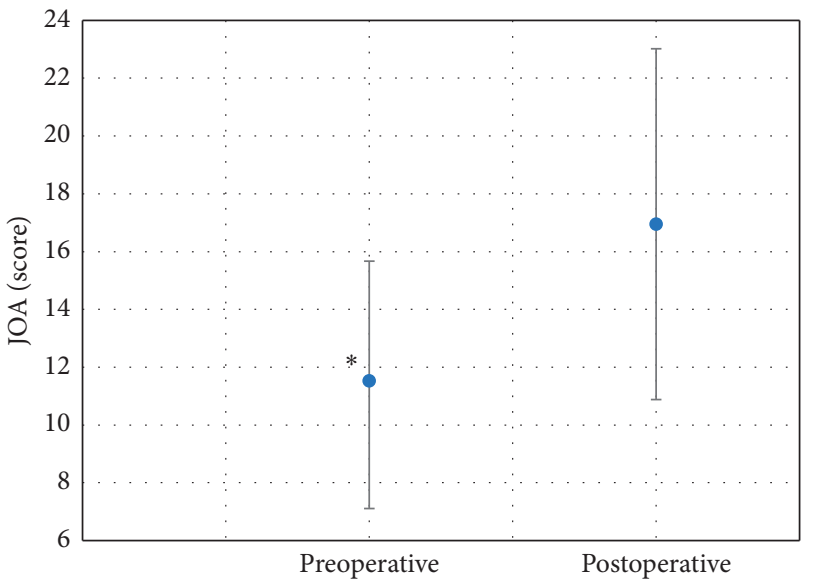

(c)

Figure 6: Comparison on C2-7Cobb, cSVA, and JOA scores of all patients before and after surgery. (a) C2-7Cobb of patients; (b) cSVA of patients; and (c) JOA of patients; ${ }^{*}$ meant that there was a statistically great difference in contrast to after surgery $(P<0.05)$.

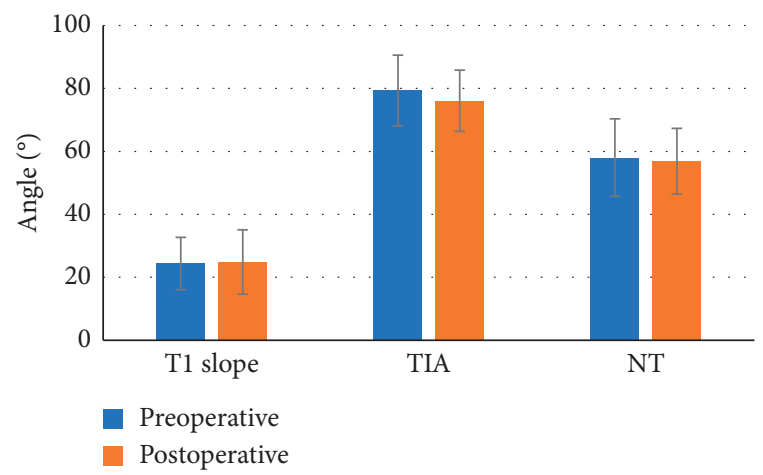

FIGURE 7: Comparison on TI slope, TIA, and NT of all patients before and after surgery.

sagittal balance. It indicates that the C2-7Cobb of patients has decreased sharply after surgical intervention, and it has approached the level of healthy people [16]. Furthermore, the CSVA and JOA scores of patients after surgery were higher greatly than those before surgery $(P<0.05)$, which also revealed that the anterior cervical spine surgery had a marked curative effect on patients.

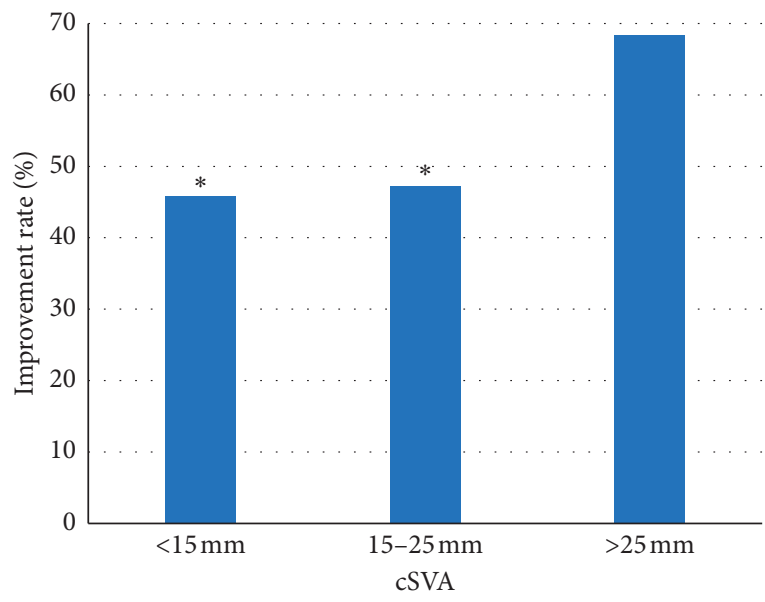

FIGURE 8: Comparison on improvement rates of patients from different cSVA groups after surgery (note: ${ }^{*}$ indicated that the difference was statistically huge in contrast to the cSVA $>25 \mathrm{~mm}$ group $(P<0.05))$.

The differences in T1 slope, TIA, and NT of patients after surgery were not statistically significant $(P>0.05)$, which was different from the research results of Liu et al. [17]. The 


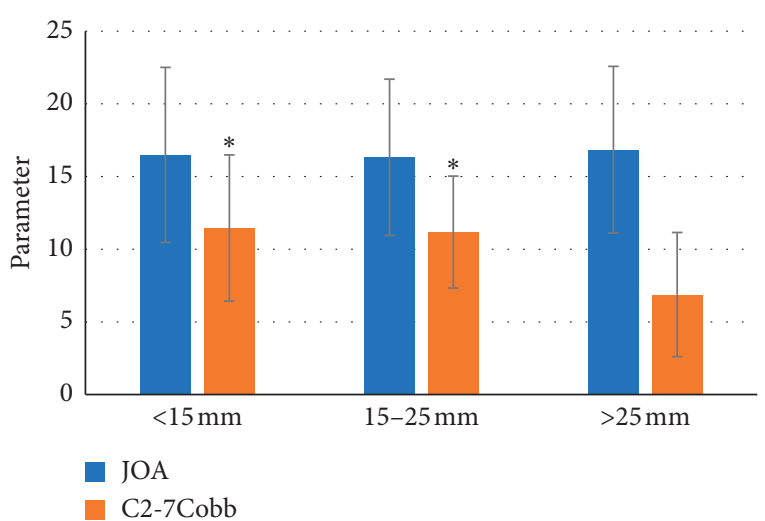

Figure 9: Comparison on C2-7Cobb and JOA scores of patients from different cSVA groups after surgery (note: * showed that the difference was statistically remarkable in contrast to the cSVA $>25 \mathrm{~mm}$ group $(P<0.05))$.

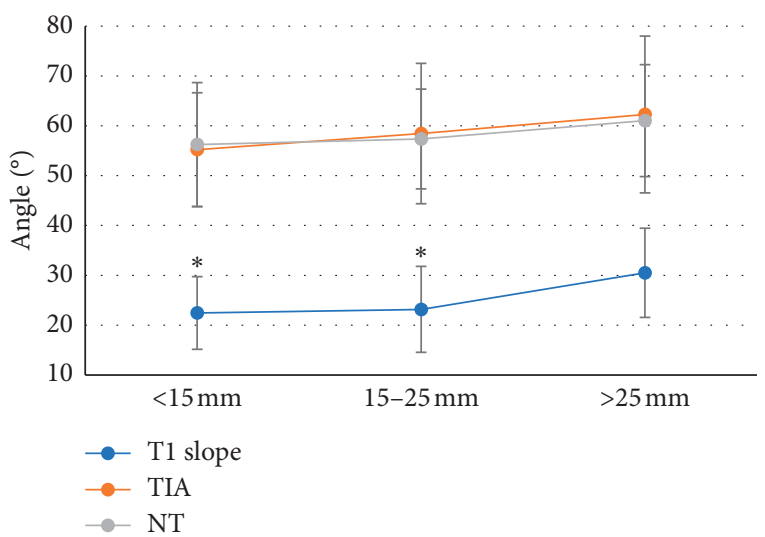

Figure 10: Comparison on T1 slope, NT, and TIA in patients from different cSVA groups after surgery (note: ${ }^{*}$ meant that the difference was statistically remarkable compared with the cSVA $>25 \mathrm{~mm}$ group $(P<0.05))$.

reason might be that the postoperative equilibrium state of patients could no longer be the same as that of healthy people, and it belonged to the irreversible equilibrium state. The improvement rate of patients from the cSVA $>25 \mathrm{~mm}$ group elevated dramatically in contrast to the rate of the other two groups $(P<0.05)$, suggesting that the anterior cervical surgery had the best improvement effect for patients with cSVA $>25 \mathrm{~mm}$. The C2-7Cobb of patients from the cSVA $>25 \mathrm{~mm}$ group dropped steeply compared with the cSVA $<15 \mathrm{~mm}$ group and the $15-25 \mathrm{~mm}$ cSVA group $(P<0.05)$, indicating that there was a negative relationship between cSVA and C2-7Cobb, namely, the greater the cSVA, the smaller the C2-7Cobb. Thus, it was speculated that the anterior cervical spine surgery should control the patient's cSVA within $25-40 \mathrm{~mm}$.

The difference in TIA of patients with cSVA $>25 \mathrm{~mm}$, cSVA $<15 \mathrm{~mm}$, and cSVA within $15-25 \mathrm{~mm}$ was not statistically huge $(P>0.05)$. This may be due to the fact that TIA is a relatively stable morphological parameter because the upper thoracic mouth is caused by the T1 vertebral body, a ring-shaped structure surrounded by ribs on both sides and the upper end of the sternum, with stable structure and low mobility [18]. Therefore, the excessive adjustment of TIA should be avoided as much as possible before the anterior cervical surgery to avoid injury to the patient. The T1 slope of patients from the cSVA $>25 \mathrm{~mm}$ group was obviously greater than that of the cSVA $<15 \mathrm{~mm}$ group and the cSVA within $15-25 \mathrm{~mm}$ group $(P<0.05)$, revealing that anterior cervical surgery had a greater adjustment to the patient's $\mathrm{T} 1$ slope.

\section{Conclusions}

The PLP-3DR was first constructed through rotation transformation and perspective projection transformation and compared with MC and GP in the performance. Then, it was employed to the X-ray film image diagnosis of 124 patients with cervical spondylosis. The results indicated that PLP-3DR proposed in this study can effectively enhance the diagnostic effect of spine X-ray images, with high sensitivity and specificity. Anterior cervical surgery had a good therapeutic effect for patients with cervical spondylosis and was closely correlated to cSVA, T1 slope, and C2-7Cobb. Moreover, patients with cSVA $>25 \mathrm{~mm}$ had the best postoperative rehabilitation effect. However, there are still some shortcomings in this study. The selected patient samples are small and from a single source. Healthy people have not been tested to obtain normal controls. The follow-up consideration is to increase the number of patients with cervical spondylosis and select healthy volunteers receiving physical examinations during the same period as a control group, thereby further exploring the three-dimensional reconstruction plan of X-ray graphics for patients undergoing cervical anterior surgeries. In summary, the results of this study can provide a good theoretical guide for the clinical treatment of anterior cervical spine surgery.

\section{Data Availability}

No data were used to support this study.

\section{Conflicts of Interest}

The authors declare no conflicts of interest.

\section{References}

[1] S. N. Salzmann, I. Okano, C. Ortiz Miller et al., "Regional bone mineral density differences measured by quantitative computed tomography in patients undergoing anterior cervical spine surgery," The Spine Journal, vol. 20, no. 7, pp. 1056-1064, 2020.

[2] S. Xu, Y. Liang, J. Wang, G. Yu, Z. Zhu, and H. Li, “Cervical spine balance of multilevel total disc replacement, hybrid surgery, and anterior cervical discectomy and fusion with a long-term follow-up," Spine (Phila Pa 1976), vol. 45, no. 16, pp. E989-E998, 2020.

[3] C. Duan, J. Hu, X. Wang, and J. Wu, "Early and one-stage posterior-anterior surgery for fresh and severe lower cervical spine fracture and dislocation," Zhong Nan Da Xue Xue Bao Yi Xue Ban, vol. 41, no. 8, pp. 838-845, 2016. 
[4] T. Liu, S. Qiu, Z. Xu et al., "Effect of anterior cervical discectomy and decompression with different fusion segments on sagittal spine-pelvis balance," Zhongguo Xiu Fu Chong Jian Wai Ke Za Zhi, vol. 33, no. 3, pp. 265-272, 2019.

[5] Y. Chen, S. Hu, H. Mao, W. Deng, and X. Gao, "Application of the best evacuation model of deep learning in the design of public structures," Image and Vision Computing, vol. 102, Article ID 103975, 2020.

[6] B. W. Burkhardt, S. J. Müller, A.-C. Wagner, and J. M. Oertel, "Anterior cervical spine surgery for the treatment of subaxial cervical spondylodiscitis: a report of 30 consecutive patients," Neurosurgical Focus, vol. 46, no. 1, p. E6, 2019.

[7] S. H. Lee, D. H. Kim, S. M. Chun, and Y.-H. Choi, "Irreversible hypoglossal nerve injury and concomitant trigeminal system dysfunction after anterior surgery to the cervical spine: case report and literature review," World Neurosurgery, vol. 136, pp. 187-192, 2020.

[8] Y. Liao, Y. Tian, R. Ye et al., "Risk and treatment of symptomatic epidural hematoma after anterior cervical spine surgery," Medicine, vol. 99, no. 2, Article ID e18711, 2020.

[9] M. S. Kang, K. H. Kim, J. Y. Park et al., "Management of esophageal and pharyngeal perforation as complications of anterior cervical spine surgery," World Neurosurgery, vol. 102, pp. 275-283, 2017.

[10] D. Armocida, G. M. F. Brunetto, L. Proietti et al., "Transoral endoscopic approach to repair early pharyngeal perforations after anterior cervical spine surgery without failure of instrumentation: our experience and review of literature," World Neurosurgery, vol. 141, pp. 219-225, 2020.

[11] R. Gamain, R. Coulomb, K. Houzir, N. Molinari, P. Kouyoumdjian, and N. Lonjon, "Anterior cervical spine surgical site infection and pharyngoesophageal perforation. Ten-year incidence in 1475 patients," Orthopaedics and Traumatology: Surgery \& Research, vol. 105, no. 4, pp. 697702, 2019.

[12] T. Rustagi, F. Alonso, D. Drazin et al., "Autologous bone harvest in anterior cervical spine surgery: a quantitative and qualitative in vitro analysis of cadaveric tissue," World Neurosurgery, vol. 112, pp. e134-e139, 2018.

[13] Y. Wu, Y. Wu, G. Gangoli et al., "Using flowable gelatin in anterior cervical spine surgery in real-world practice: a retrospective cohort study," Journal of Comparative Effectiveness Research, vol. 8, no. 5, pp. 317-326, 2019.

[14] J. Rama-López, M. Tomás-Fernandez, C. García-Garza, and M. Martínez-Madrigal, "Pharyngeal perforation after anterior cervical spine surgery treated by transoral endoscopic surgery," Head \& Neck, vol. 40, no. 2, pp. E13-E16, 2018.

[15] $\varnothing$. Helseth, B. Lied, B. Heskestad, K. Ekseth, and E. Helseth, "Retrospective single-centre series of 1300 consecutive cases of outpatient cervical spine surgery: complications, hospital readmissions, and reoperations," British Journal of Neurosurgery, vol. 33, no. 6, pp. 613-619, 2019.

[16] C. Simon, C. H. Fürstenberg, M. Eichler, S. Rohde, C. Bulut, and B. Wiedenhöfer, "Management of pharyngeal fistulas after anterior cervical spine surgery," Clinical Spine Surgery: A Spine Publication, vol. 30, no. 1, pp. E25-E30, 2017.

[17] J. Liu, Y. Hai, N. Kang, X. Chen, and Y. Zhang, "Risk factors and preventative measures of early and persistent dysphagia after anterior cervical spine surgery: a systematic review," European Spine Journal, vol. 27, no. 6, pp. 1209-1218, 2018.

[18] S. K. Kim, J. B. Park, J. Y. Chung et al., "Anterior cervical debridement and fusion for cervical pyogenic spondylodiscitis: use of anterior cervical plating or not?" Spine (Phila Pa 1976), vol. 45, no. 7, pp. 431-437, 2020. 